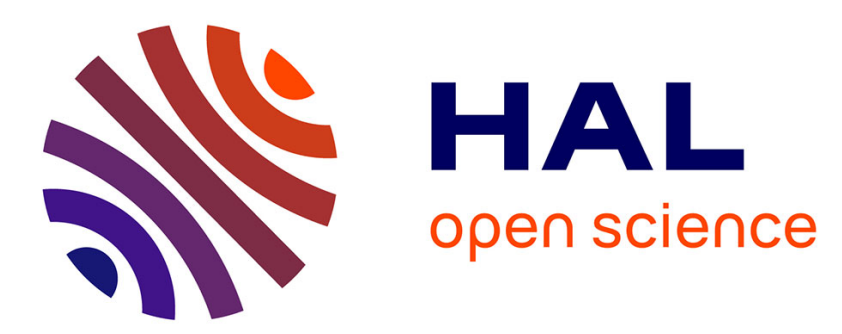

\title{
Two Node-Disjoint Paths Routing for Energy-Efficiency and Network Reliability
}

\author{
Rihab Abid Maaloul, Raouia Taktak, Lamia Chaari, Bernard Cousin
}

\section{To cite this version:}

Rihab Abid Maaloul, Raouia Taktak, Lamia Chaari, Bernard Cousin. Two Node-Disjoint Paths Routing for Energy-Efficiency and Network Reliability. 25th International Conference on Telecommunication (ICT 2018), Jul 2018, Saint-Malo, France. pp.554 - 560, 10.1109/ICT.2018.8464840 . hal-01875381

\section{HAL Id: hal-01875381 \\ https://hal.science/hal-01875381}

Submitted on 17 Sep 2018

HAL is a multi-disciplinary open access archive for the deposit and dissemination of scientific research documents, whether they are published or not. The documents may come from teaching and research institutions in France or abroad, or from public or private research centers.
L'archive ouverte pluridisciplinaire HAL, est destinée au dépôt et à la diffusion de documents scientifiques de niveau recherche, publiés ou non, émanant des établissements d'enseignement et de recherche français ou étrangers, des laboratoires publics ou privés. 


\title{
Two Node-Disjoint Paths Routing for Energy-Efficiency and Network Reliability
}

\author{
Rihab Maaloul*, Raouia Taktak* Lamia Chaari*and Bernard Cousin ${ }^{\dagger}$ \\ *Laboratory of Technology and Smart Systems (LT2S), \\ Digital Research Center of SFAX (CRNS), University of Sfax, Tunisia \\ $\dagger$ University of Rennes 1, IRISA, France
}

\begin{abstract}
Carrier grade networks are in general overdimensioned in order to protect against network resource failures and to handle probable traffic peaks. Such network design exhibits non-negligible energy waste. As well known, during network operation, the traffic load varies remarkably over day hours. Typically, a significant amount of energy saving can be achieved by turning off redundant and underutilized resources. Ensuring a sufficient level of reliability while taking into account energy saving objective is a very challenging task. In this paper, we study the multi-commodity reliable network design for carrier Ethernet networks. Each traffic demand is routed along one working path, and a corresponding backup path computed considering dedicated protection scheme. The primary and backup path must be node-disjoint. We also assume that the links of the carrier grade networks are made of multiple physical cables called bundles. We propose an exact method based on an Integer Linear Programming (ILP) formulation for the two node-disjoint paths with energy-aware routing (TNDPEAR) problem. The studied problem is known to be NP-hard. In order to solve it efficiently, we propose a heuristic-based algorithm called GreenTNDP. Preliminary experimental results show the effectiveness of our algorithms to solve the problem.
\end{abstract}

Keywords-Energy-aware routing; Two disjoint paths; Carrier grade networks; Reliability; Network optimization

\section{Introduction}

Reducing electricity bills and energy consumption has become a crucial goal for all industries, including telecommunication operators and Internet Service Providers (ISP). At the moment, network operators tend simultaneously to optimize the energy expenditure of their network and to provide the high service level required by their users. However, these two objectives are contentious. A tradeoff between both becomes hence inevitable. As the traffic demand continues to grow, it requires additional network resources with higher capacities and faster processing speeds. On the other hand, some critical applications need to be protected against unexpected failure events. Additional resources and power are consequently needed when it comes to ensuring network reliability. Network resilience versus energy-efficiency is a key concern in carrier grade networks [1], [2]. The terms 'resilience', 'survivability', and 'reliability' are interchangeably used in the literature of telecommunications networks to refer to networks that are able to survive in the face of different faults. Network resilience implementation implies a mechanism for fault detection and localization together with a set of recovery techniques to reroute traffic around the failed component.
The path used by default is called working path or primary path. The path used to replace the working path in case of failure is called backup path. Depending on whether backup paths are computed before or after a fault of the working path, recovery techniques can be implemented based on two general schemes, restoration or protection [1]. Routing with node-disjoint backup paths is more resilient to failures than routing with link-disjoint backup paths, because it can protect against both node and link failures. Furthermore, routing with node-disjoint paths is also link-disjoint, but not vice versa.

For network devices (i.e., carrier Ethernet devices) that present ON-OFF power profile [3], [4], energy savings can be achieved by turning off selected devices (enter into sleep mode). To this end, many energy-aware routing solutions have been proposed [5]-[10] to route traffic over energy efficient paths, and turn off unused network elements. Of particular interest are approaches such as [5] and [7] which propose an energy-aware routing with two disjoint paths maintaining the network reliability.

In addition, bundled link concept can help improve the network capacity along with an enhanced resilience in case of link failure. This technique of composite link, called also link aggregation, is standardized by the IEEE 802.1AX [11]. Two main reasons behind using bundled links are (i) allowing network operators to easily upgrade their network capacity, (ii) resilience in case of cable failures and congestion. In this paper, a single network link is referred to one bundled link that is composed of multiple cables (or ports). We assume, without loss of generality, that all the cables have the same capacity and the same power consumption, and each port can be turned off independently.

In this paper, we consider the problem called energyaware routing with two node-disjoint reliable paths (TNDP-EAR). To the best of our knowledge, this is the first work that jointly minimizes the number of active cables in bundled links whilst ensuring a two-node disjunction protection scheme. To tackle this problem, we use efficient optimization techniques. Our main contributions are:

- First, we model the TNDP-EAR problem as an integer linear program. This exact formulation provides optimal solutions for small networks.

- Second, we propose a heuristic-based method called GreenTNDP, suitable for large-sized networks. 
The paper is organized as follows. We present related works in the next section. In Section III, we formally describe the problem and model it as an ILP formulation. In Section IV, we describe our heuristic algorithm. A performance analysis of the proposed resolution methods is presented in Section V. Finally, we give concluding remarks in Section VI.

\section{Related Works}

The first work proposed in the context of green networking was that of Gupta et al. [12], where a network protocol is able to put network components to sleep. They propose coordinated sleeping solution that deals with routing protocol aggregating traffic into few routes, in order to enable some links to sleep. Chiaraviglio et al. [9] provide a basic formulation of the EAR problem as a capacitated multi-commoditiy flow (CMCF) problem. They also propose different heuristics based on several sorting policies to turn off both links and nodes. Capone, et al. [13] propose an optimization model that aims at minimizing the energy consumption of nodes and links while considering load-balancing constraints. The main shortcoming of this approach is the use of the traditional Multiple Spanning Tree Protocol (MSTP) [14], which can not adhere any more to the needs of nowadays carrier Ethernet network. Fisher et al. [10] were the first who consider that pairs of core routers are connected by bundled links consisting of multiple cables. They propose three heuristic solutions, based on linear relaxation, to quickly determine the sub-optimal set of cables to be turned off. Their heuristics reroute traffic demands in a splittable way, i.e., multiple paths can be used to transmit one traffic demand between a source and a destination. In another work [15], the same problem is addressed but with single path routing, i.e., traffic demands are routed in unsplittable way. Computation of the k-shortest-paths is exploited to select the path routing which fulfills the maximum link utilization constraint.

In addition to energy efficiency, many studies have been interested to reliability issues in the network design problems. In [16] and [17], the authors provide a set of algorithms and optimization models suitable with IP networks, considering unsplittable routing. In both works, the network resilience requirements are taken into account while reducing the link and node consumption. In addition to network survivability, robust optimization model is proposed in [17] to handle uncertain traffic demands. In [7], Lin et al. have proposed an energy-aware routing with two disjoint paths, according to which each request can be routed via multiple paths (which may have common links) besides two disjoint paths, to provide higher protection against eventual failures. The same authors have proposed an interesting strategy [6] which considers reliability and protection constraints in the formulation. The authors propose a comparative study of green routing approaches using two reliability measures: Terminal Reliability (TR) and Route Reliability (RR). Finding primary and backup link-disjoint paths for each source-to-terminal request is proposed in [5]. Two heuristic algorithms based on the computation of Suurballe's algorithm [18] to find the working and backup paths, while turningoff both nodes and links are presented and evaluated. The optimization model aims at minimizing the fixed energy cost paid to keep a router or a link in the active state. To obtain only a single active path routing, binary flow variables are introduced. Note that the solution proposed in [5] can protect against only link failures, i.e., it does not consider the node failure event. To the best of our knowledge, the closest works to ours are [5]-[7]. Like ours, they all are intended to be run in a logically centralized manner. We report in TABLE I the main common and different points (assumptions and resolution method) between our work, and those of [5], [6] and [7].

\section{Problem formulation}

\section{A. Basic assumptions and notations}

Our network model is based on the following assumptions: (i) each link consists of $B$ cables that can be turned off independently, we assume to turn off only cables. (ii) we consider that for any pair of network nodes, it is possible to compute at least two node-disjoint paths. (iii) single link/node failure (the probability of simultaneous failures is very rare because failures are very quickly solved).

Let $G=(V, A)$ be a directed graph that model the network topology, where $V$ is the set of nodes (Ethernet switches), and $A$ is the set of links. Each link $(u, v)$ represents a bundled link with $B_{u v} \geqslant 1$ cables. The capacity of each link, i.e., $C_{u v} \geqslant 1$, is the total capacity of its cables, and each cable has the same capacity. The power consumption of each link is the total consumption induced by its operational cables. The number of turned-on cables in any given link $(u, v)$ is denoted $n_{u v} \leqslant B_{u v}$. When $n_{u v}=0$, it means that the whole link $(u, v)$ is turned off. Let $D$ be the set of all traffic demands (traffic matrix) in $G$, and the triplet $\left(s_{d}, t_{d}, h_{d}\right)$ denote the traffic demand indexed by $d=1,2, \ldots,|D|$ from source node $s_{d} \in V$ to destination node $t_{d} \in V$, where $h_{d}$ is the amount of traffic exchanged from $s_{d} \in V$ to $t_{d} \in V$.

Given $G$ and $D$ as input, the TNDP-EAR (Two Node Disjoint Paths energy-aware routing) problem consists in routing each demand in an unsplittable way through a primary path $P_{p}^{d}$ and a backup path $P_{b}^{d}$, while minimizing the number of powered-on cables. Additionally, the routing solution has to respect the maximum link utilization rate threshold that is denoted as $U_{T}$. We denote by $f_{u v}$ the total flow assigned to link $(u, v) \in A, y_{u v}^{d}$ the flow of $d$ that is routed on link $(u, v)$ used by primary path $P_{p}^{d}$, and $z_{u v}^{d}$ the flow of $d$ that is routed on link $(u, v)$ used by backup path $P_{b}^{d}$. The link utilization of link $(u, v)$ is calculated as $\mu_{u v}=100 \times\left(f_{u v} / C_{u v}\right)$. Finally, $g_{u}^{d}$ and $k_{u}^{d}$ are binary variables that indicate whether node $u$ is used to route $d$ through the primary path $P_{p}^{d}$ or the backup path $P_{b}^{d}$ respectively. TABLE II summarizes all the mentioned 
TABLE I: Main properties of this study with respect to the closest proposals

\begin{tabular}{|c|c|c|c|c|}
\hline Properties & {$[5]$} & {$[6]$} & {$[7]$} & This work \\
\hline Routing & Unsplittable & Free splittable & Splittable & Unsplittable \\
\hline Path computation & Suurballe's algorithm [18] & Yen's algorithm [19] & Yen's algorithm [19] & Basic disjoint path algorithm [20] \\
\hline Exact formulation & -1 ILP & MILP & MILP & ILP \\
\hline Backup paths & Dedicated & Dedicated and shared & Dedicated and shared & Dedicated \\
\hline Failure assumption & Link & Link & Node and link & Node and link \\
\hline Bundled links & - & $\checkmark$ & - & $\checkmark$ \\
\hline
\end{tabular}

notations for the parameters and decision variables.

To illustrate the TNDP-EAR problem, we consider an example of 6-nodes network as shown in Fig. 1. We assume that each link is made of four cables $(B=4)$. The capacity of each bundled link is assumed to be 24 units. Assume that there are two demands on the network, i.e., $D=$ $\{(0,2,2),(0,5,3)\}$. Fig. 1b presents an optimal solution for TNDP-EAR problem. For demand $d=1$, the primary path $P_{p}^{1}=0 \rightarrow 2$, and the backup path $P_{b}^{1}=0 \rightarrow 1 \rightarrow 3 \rightarrow 2$. For demand $d=2$, the primary path $P_{p}^{2}=0 \rightarrow 2 \rightarrow 4 \rightarrow$ 5 , and the backup path $P_{b}^{2}=0 \rightarrow 1 \rightarrow 3 \rightarrow 5$. As we consider $1+1$ protection, the traffic is duplicated over the two disjoint paths. In this case, we obtain $f_{01}=f_{02}=f_{13}=$ $5 ; f_{32}=2 ; f_{35}=f_{24}=f_{45}=3$. Dashed lines correspond to links that are totally turned off, whereas turned off cables are not illustrated. As the spare capacity on each arc can be turned off by rounding up to discrete number of cables, we obtain $n_{01}=n_{02}=n_{13}=2 ; n_{32}=n_{35}=n_{24}=n_{45}=1$; $n_{14}=n_{34}=0$.

Fig. 1c illustrates the TNDP-EAR solution in case of node 4 failure. Demand $d=1$ keeps its both paths while demand $d=2$ is served along $P_{b}^{2}$.

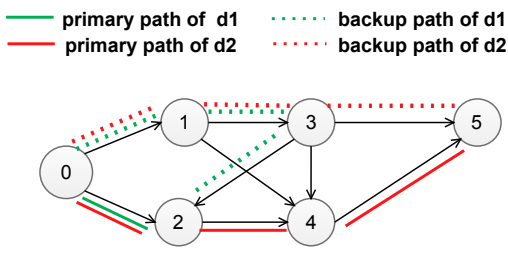

(a) Network topology before solving TNDP-EAR problem

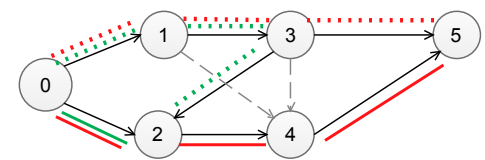

(b) Routing solution for TNDP-EAR (no failure)

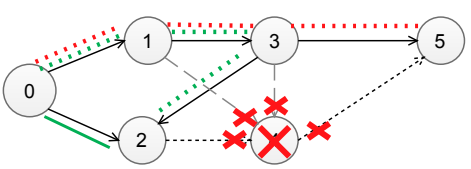

(c) Routing solution for TNDP-EAR (node 4 failure)

Fig. 1: Example of a routing solution for TNDP-EAR problem
B. The integer programming model

We formulate the TNDP-EAR problem as an integer linear programming model for directed graphs as follows.

$$
\begin{array}{cr}
\min \sum_{(u, v) \in A} n_{u v} \mathcal{E}_{u v}+\beta\left\{\sum_{(u, v) \in A}\left(B_{u v}-n_{u v}\right) \mathcal{E}_{u v}\right\} \\
x_{u v} \leq n_{u v} & \forall(u, v) \in A, \\
B_{u v} x_{u v} \geq n_{u v} & \forall(u, v) \in A,
\end{array}
$$

$\sum_{(u, v) \in A} y_{u v}^{d}-\sum_{(v, u) \in A} y_{v u}^{d}=\left\{\begin{array}{ll}1 & \text { if } u=s_{d} \\ -1 & \text { if } u=t_{d} \\ 0 & \text { if } \mathrm{u} \neq s_{d}, t_{d}\end{array} \quad \forall d \in D\right.$

$$
\sum_{(u, v) \in A} z_{u v}^{d}-\sum_{(v, u) \in A} z_{v u}^{d}=\left\{\begin{array}{ll}
1 & \text { if } u=s_{d} \\
-1 & \text { if } u=t_{d} \\
0 & \text { if } \mathrm{u} \neq s_{d}, t_{d}
\end{array} \quad \forall d \in D\right.
$$$$
\sum_{d \in D} h_{d}\left(y_{u v}^{d}+z_{u v}^{d}\right) \leq U_{T}\left(n_{u v} / B_{u v}\right) C_{u v} \forall(u, v) \in A,
$$

$$
\begin{gathered}
y_{u v}^{d}+z_{u v}^{d} \leq x_{u v} \quad \forall(u, v) \in A ; d \in D, \\
g_{u}^{d}+k_{u}^{d} \leq 1 \quad \forall u \in V \backslash\left\{s_{d}, t_{d}\right\} ; d \in D, \\
y_{u v}^{d}+y_{v u}^{d} \leq g_{u}^{d} \quad \forall u \in V ;(u, v) \in A ; d \in D, \\
z_{u v}^{d}+z_{v u}^{d} \leq k_{u}^{d} \quad \forall u \in V ;(u, v),(v, u) \in A ; d \in D .
\end{gathered}
$$

The objective function (1) minimizes the total power consumption induced by cables. It is composed of two parts. The first part computes the power consumption of powered on cables. The second part computes the consumption of powered-off cables. It is weighted by parameter $\beta$ that is set to 0.1 , assuming that the powered-off cables consume $10 \%$ of the power spent in the active mode. Inequalities (2) make sure that all cables of link $(u, v)$ are powered-off when $x_{u v}=0$. Inequalities (3) make sure if link $(u, v)$ has at least one cable powered-on (i.e., $n_{u v} \geq 1$ ) then $x_{u v}=1$. Equations (4) and (5) express for each demand the classical flow conservation constraints for the primary and backup paths, respectively. Constraints (6) ensure that the total flow going through each link $(u, v)$ of the primary and backup path cannot exceed the tolerated link capacity, i.e., $U_{T}\left(n_{u v} / B_{u v}\right) C_{u v}$. Inequalities (7) make sure that the primary and backup paths are link disjoint. While, inequalities (8) guarantee that for each demand the primary and the backup paths are node-disjoint. Finally, inequalities (9) and (10) are for two node-disjoint paths. They guarantee that if node $u$ is used by primary path 
TABLE II: Summary of notations and parameters

\begin{tabular}{|c|c|}
\hline Notation & Description \\
\hline $\mathrm{G}=(V, A)$ & Directed graph where $V$ is the set of vertices (nodes) and $A$ is the set of arcs (links) \\
\hline $\mathrm{G}^{\prime}=\left(V, A^{\prime}\right)$ & graph solution used to route $D$ \\
\hline $\mathcal{E}_{u v}$ & Power consumption of a powered cable in link $(u, v) \in A$ \\
\hline$\beta$ & Carameter set to 0.1, assuming that a powered-off cables consumes $10 \%$ of the power spent in the active mode \\
\hline$C_{u v}$ & Capacity of link $(u, v) \in A$ \\
\hline$U_{T}$ & Remaining capacity of link $(u, v)$ \\
\hline$r_{u v}$ & Set of all traffic demands $D=\left\{\left(s_{d}, t_{d}, h_{d}\right), s_{d} \in V, t_{d} \in V\right\}$ \\
\hline$D$ & Set of all demands having t as destination node $t \in V$ \\
\hline$D^{t}$ & Demand of the traffic flow from node $s_{d}$ to $t_{d}$ \\
\hline$h_{d}$ & Number of cables in link $(u, v) \in A$ \\
\hline$B_{u v}$ & Integer variable to indicate the number of powered-on cables in link $(u, v)$ \\
\hline$n_{u v}$ & Binary variable to indicate if the link $(u, v)$ has at least one powered-on cable or not \\
\hline$x_{u v}$ & Binary variable to indicate whether link $(u, v)$ is used to route $d$ by primary path $P_{p}^{d}$ \\
\hline$y_{u v}^{d}$ & Binary variable to indicate whether link $(u, v)$ is used to route $d$ by backup path $P_{b}^{d}$ \\
\hline$z_{u v}^{d}$ & Total flow of link $(u, v) ; f_{u v} \geq 0$ \\
\hline$f_{u v}$ & Binary variable to indicate whether node $u$ is used to route $d$ by primary path $P_{p}^{d}, u \in V \backslash\left\{s_{d}, t_{d}\right\}$ \\
\hline$g_{u}^{d}$ & \multicolumn{2}{c}{ bariable to indicate whether node $u$ is used to route $d$ by backup path $P_{b}^{d}, u \in V \backslash\left\{s_{d}, t_{d}\right\}$} \\
\hline$k_{u}^{d}$ &
\end{tabular}

$\left(g_{u v}^{d}=1\right)$, then node $u$ should be excluded from the backup path $\left(k_{u v}^{d}=0\right)$, and vice versa.

\section{Heuristic algorithm}

As the EAR link disjoint path is proven in [5] to be NP-hard even for only two different demands. Thus, we can conclude that TNDP-EAR problem is an NP-hard problem as well. Hence, heuristic methods are preferred to quickly find efficient solutions for this problem. We propose, GreenTNDP, a heuristic algorithm that includes a nodedisjoint path computation phase and an EAR phase. The implementation of GreenTNDP can be run on a logicallycentralized power monitor such as in Software Defined Network (SDN) architecture. The idea of our heuristic is as follows (see Algorithm 1). Sort all demands in a descending order of their traffic $h_{d}$; create the residual graph for each demand by removing all links with residual capacity lower than $h_{d}$; find the primary path and backup path (using algorithm 2 that computes node-disjoint paths [20]) for each demand. Then, the energy aware routing phase is initialized; select iteratively a link with maximum residual capacity to turn off; find all demands that contain the deleted link and reroute traffic for affected demands. If any of the affected demands fails to be rerouted, the candidate link must be turned on. Repeat the process until all links are considered. Finally, turn-off the unused cables using (11). The GreenTNDP heuristic runs in a polynomial time and has a complexity of $O\left(|A|^{2}+|A||V| \log (|V|)\right)$.

$$
n_{u v}=\left\lceil\frac{f_{u v} B_{u v}}{\mu C_{u v}}\right\rceil
$$

\section{Experimental results}

In this section, we evaluate the flow based ILP formulation for directed graphs and the heuristic-based algorithm GreenTNDP.

We use the following experiments setup (i) bundle size $B_{u v}=4 ;$ (ii) the maximum tolerated link utilization $U_{T}=100 \%$; and (iii) each cable has the same power consumption $\mathcal{E}_{u v}=100$ watts.
Input: A weighted-directed graph $G=(V, A)$, a set $D$ of demands with traffic requirements $h_{d}$ for all $d \in D$,

Output: $G^{\prime}=\left(V, A^{\prime}\right)$ : the output graph containing only links used to route the demands.

1 Initially, $A^{\prime}=A$;

$2 /{ }^{*}$ Phase $1 * /$ Two Node Disjoint Path

3 Paths $=\emptyset$;

4 Sort demands in a descending order of traffic $h_{d}$;

5 for each $d \in D$ do

$6 \quad$ Compute the residual graph $G_{r}$ for $d$ by removing the links with $r_{u v} \leq h_{d}, \forall(u, v) \in A^{\prime}$;

$P_{d}=$ call find-TNDP $\left(G_{r}, d\right) \quad / *$ Algorithm $2^{*} /$;

if $P_{d}$ exist then

Update residual capacity of links of $P_{d}$ on $G^{\prime}$; Paths $=$ Paths $\cup P_{d} ; P_{d}=\left\{P_{p}^{d}, P_{b}^{d}\right\}$;

return true;

else

return false;

break ;

end

6 end

$7 / *$ Phase 2*/ Energy Aware Routing

$8 / /$ Step 1

9 for each link $(u, v) \in A^{\prime}$ in a descending order of its residual capacity do

$A^{\prime}=A^{\prime}-\{(u, v)\} ; / /$ turn off candidate link $(u, v)$

$/ /$ Find affected demands by turning off $(u, v)$

$D^{a}=\left\{d \mid P_{d} \cap(u, v) \neq \emptyset, d \in D\right\} ;$

Release the bandwidth of $D^{a}$

feasible=Reroute traffic of $D^{a}$ on $G^{\prime}$ by repeating

Phase 1;

if feasible $==$ false then

put $(u, v)$ back to $A^{\prime}$;

assign bandwidth of $D^{a}$ on $G^{\prime}$;

end

end

//Step 2

for each each link $(u, v) \in A^{\prime}$ do

$\$ 2$ turn off unused cables using (11);

3 end

Algorithm 1: Pseudo-code description for the proposed heuristic (GreenTNDP)

To evaluate the performance of our algorithms, we use the following metrics. 
Input: A weighted-directed graph $G=(V, A)$, a pair of source and destination nodes $(s, t)$.

Output: 2 node-disjoint paths $P_{1}, P_{2}$.

1 for $\mathrm{i}=1,2$ do

$2 \quad$ Find the shortest path $P_{i}$ from node $s$ to node $t$; 4 end

Delete intermediate node of $P_{i}$ from $G$;

Algorithm 2: Basic 2-node-disjoint-paths in directed graphs [20]

$\eta \%$ is the percentage of power savings related to the cables turned off by the EAR algorithm. It is computed as follows:

$$
\eta \%=\left(1-\frac{\sum_{(u, v) \in A} n_{u v}}{\sum_{(u, v) \in A} B_{u v}}\right) \times 100 \% .
$$

$\rho$ is used to measure the mean traffic utilization of the used links in $G^{\prime}$.

$$
\rho \%=100 \times \frac{\sum_{(u, v) \in A^{\prime}} \rho_{u v}}{\left|A^{\prime}\right|},
$$

where $\rho_{u v}$ is the traffic utilization of link $u v$.

Finally, we evaluate the effect of our EAR algorithms according to the reliability of each demand. We measure route reliability as in [6]. Let $p_{u v}=0.9$ be the probability of a cable in link $(u, v)$ being functional. The reliability of a link $(u, v)$ denoted by $l_{u v}$ is computed as follow:

$$
l_{u v}=1-\left(1-p_{u v}\right)^{n_{u v}} .
$$

Let $R R_{d}$ denote the global route reliability for a demand $d$ routed through two node-disjoint paths (i.e., represented by variables $y$ and $z$ ). $R R_{d}$ is computed as follows:

$$
R R_{d}=1-\left(1-R_{d y}\right)\left(1-R_{d z}\right),
$$

where $R_{d y}$ and $R_{d z}$ are the route reliability of the primary and back-up paths respectively. $R_{d y}$ and $R_{d z}$ are calculated by the following formula :

$$
R_{d\{y / z\}}=\log ^{-1}\left(\sum_{(u v) \in R_{d\{y / z\}}} \log l_{u v}\right), \forall d \in D .
$$

We can also compute the average route reliability (ARR) for a given traffic matrix $D$ as follows.

$$
A R R=\frac{\sum_{d \in D} R R_{d}}{|D|} .
$$

We test our algorithms the ILP and GreenTNDP on six network topologies collected from the SNDlib [21]. We choose network instances where two node-disjoint paths always exist. We solve the ILP model using the CPLEX solver [22], with a time limit set to 3 hours. Cplex is used for a branch-and-bound resolution, cuts are deactivated. TABLE III and TABLE IV report the computational results. The gap column reports the energy performance of the resulting network topology, if the gap equals to zero, it means that the optimal solution was found.
The obtained results confirm the effectiveness of our heuristic GreenTNDP. For instance, the ILP model cannot find an optimal solution for Atlanta, Dfn-bwin, Di-yuan, and Nobel-germany networks within 3 hours, meanwhile GreenTNDP provides feasible solutions in reasonable time (within at most 11 seconds). Moreover, we notice that the heuristic algorithm outperforms the ILP model for Atlanta, Di-yuan, and Nobel-germany instances, not only in terms of computational time but also in terms of energy savings. For Atlanta $73 \%$ of cables are turned off in 19.31 seconds by GreenTNDP, whereas $67 \%$ of cables are turned off in 3 hours by the ILP. Concerning Pdh network instance, ILP model and GreenTNDP find the same solution (optimal). This result shows that GreenTNDP may reduce the computation time. Finally, ILP model can find the optimal solution for Polska instance within 1529 seconds. Meanwhile GreenTNDP find feasible solution (with $19.43 \%$ of gap to optimality) in only 6.86 seconds. We can hence conclude that our heuristic should be improved in order to get approximative solutions close to the optimal ones.

Note that once some of the links are turned-off, the same traffic is rerouted on a fewer set of links, which impact the traffic capabilities (i.e., network congestion). This trade-off between energy-saving and network congestion is clearly demonstrated by $\rho$ parameter. We notice that the mean link utilization $\rho \%$ increases when $\eta \%$ increases as well. For example, when evaluated on Atlanta, the ILP can turn off $67.6 \%$ of cables having a smaller impact on the mean traffic utilization ( $\rho=23 \%)$, compared to $(\rho=52.72 \%)$ when GreenTNDP achieved higher energy saving, i.e., $\eta=73.29$. Figures 2 to 4 present the topologies before and after the execution of Green TNDP for Atlanta, Dfn-bwin and Diyuan network instances.

TABLE $\mathrm{V}$ highlights the trade-off between minimizing energy usage and enhancing network reliability. We show the route reliability for GreenTNDP, ILP model and SP (Shortest Path routing without disjoint backup path) for the six network instances. Note that SP is an energy aware routing that minimizes the total number of cables that route each demand along only one path (the primary one). The results inTABLE $\mathrm{V}$ show that ILP model and GreenTNDP produce topologies with highest reliability (ARR) as compared to SP, since this later uses a fewer number of paths to route the demands. In the other hand, we observe that ARR increases when $\eta \%$ decreases for all the algorithms. These results are expected because when rerouting traffic on fewer cables this increases (resp. decreases) energy saving (resp. route reliability).

\section{Conclusion}

To the best of our knowledge, this is the first study that deals with the energy-aware routing with two nodedisjoint paths problem. In this paper, we formally defined the reliable energy-aware routing problem and modeled it using an ILP formulation. We then proposed a greedy heuristic algorithm GreenTNDP. Our evaluation of the ILP model TNDP-EAR and the heuristic GreenTNDPbasic on six realistic networks showed that the heuristic is efficient 
TABLE III: ILP formulation

\begin{tabular}{|cccc|c|c|c|c|c|}
\hline Network & $|V|$ & $|E|$ & $|D|$ & $\begin{array}{c}\text { Power } \\
\text { Saving } \\
(\eta \%)\end{array}$ & $\begin{array}{c}\text { Optimality } \\
\text { gap } \\
(\%)\end{array}$ & $\begin{array}{c}\text { Power consumption } \\
\text { Upper bound } \\
\text { UB }(\mathrm{W})\end{array}$ & $\begin{array}{c}\text { Mean links } \\
\text { Utilization } \\
(\rho)\end{array}$ & $\begin{array}{c}\text { Execution } \\
\text { Time } \\
(\mathrm{s})\end{array}$ \\
\hline Atlanta & 15 & 44 & 132 & 67.61 & 5 & 5147.6 & 23.29 & 10800 \\
\hline Dfn-bwin & 10 & 90 & 90 & 90.83 & 21 & 3006 & 22.46 & 10800 \\
\hline Di-yuan & 11 & 84 & 22 & 88.39 & 6 & 3543.6 & 22.09 & 10800 \\
\hline Nobel-germany & 17 & 52 & 121 & 55.28 & 2 & 8390.8 & 43.89 & 10800 \\
\hline Pdh & 11 & 68 & 24 & 88.06 & 0 & 1917.2 & 11.05 & 30.25 \\
\hline Polska & 12 & 36 & 66 & 71.52 & 0 & 3704.4 & 27.66 & 1529.89 \\
\hline
\end{tabular}

TABLE IV: Heuristic algorithm (GreenTNDP)

\begin{tabular}{|cccc|c|c|c|c|}
\hline Network & $|V|$ & $|E|$ & $|D|$ & $\begin{array}{c}\text { Power } \\
\text { Saving } \\
(\eta \%)\end{array}$ & $\begin{array}{c}\text { Power consumption } \\
\text { Upper bound } \\
\text { UB }(\mathrm{W})\end{array}$ & $\begin{array}{c}\text { Mean links } \\
\text { Utilization } \\
(\rho)\end{array}$ & $\begin{array}{c}\text { Execution } \\
\text { Time } \\
(\mathrm{s})\end{array}$ \\
\hline Atlanta & 15 & 44 & 132 & 73.29 & 4247.6 & 52.72 & 19.31 \\
\hline Dfn-bwin & 10 & 90 & 90 & 87.22 & 4176.0 & 25.32 & 31.92 \\
\hline Di-yuan & 11 & 84 & 22 & 91.66 & 2553.6 & 11.94 & 8.96 \\
\hline Nobel-germany & 17 & 52 & 121 & 75 & 5061.8 & 13.90 & 30.54 \\
\hline Pdh & 11 & 68 & 24 & 88.06 & 1917.2 & 11.05 & 8.20 \\
\hline Polska & 12 & 36 & 66 & 65.97 & 4424.4 & 18.84 & 6.86 \\
\hline
\end{tabular}

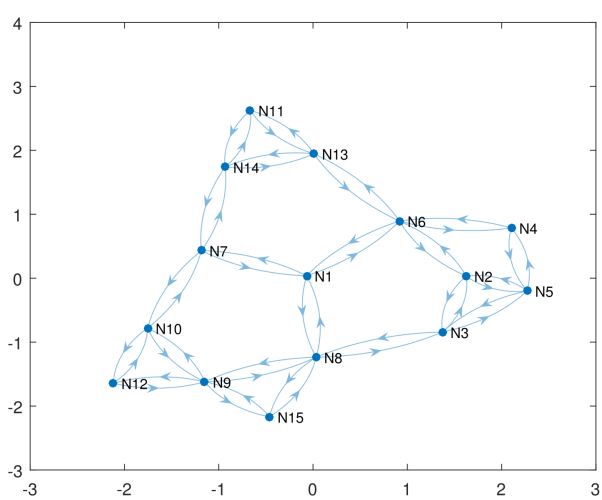

(a) Before GreenTNDP execution

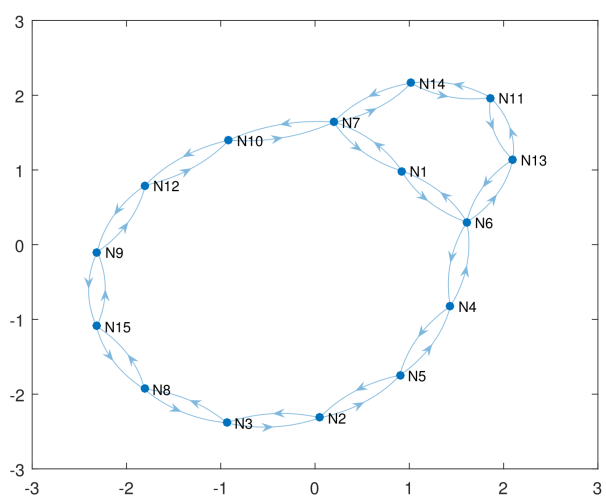

(b) After GreenTNDP execution

Fig. 2: Atlanta network

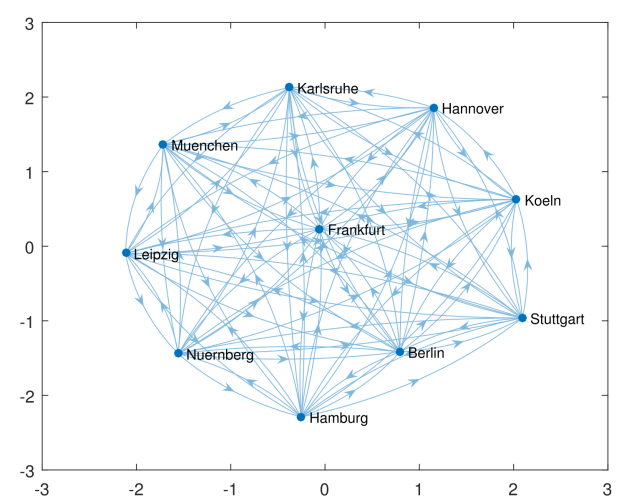

(a) Before GreenTNDP execution

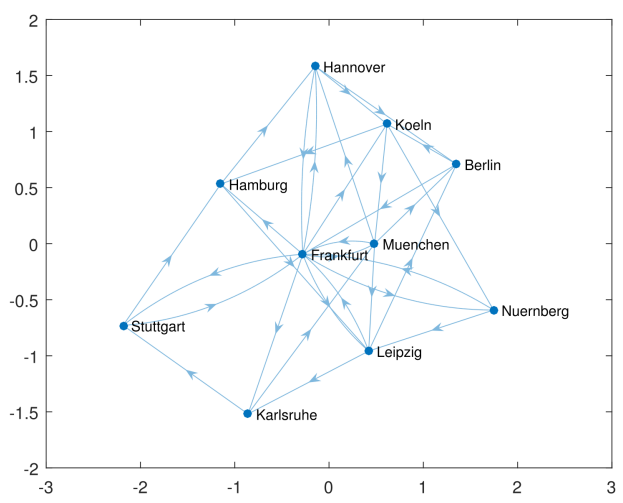

(b) After GreenTNDP execution

Fig. 3: Dfn-bwin network to produce a network with a low energy usage under a high-reliability level in reasonable time. However, several improvements would be interesting to consider in order to enhance the heuristic algorithm and get close-to-optimal solutions. In this context, a theoretical analysis of the bounds of the heuristic algorithm could be considered in the future. As a future work, we aim at comparing the performances of our algorithms to the literature ones (i.e., [5]-[7]), mainly considering the link-disjunction along with node-disjunction. Recall also that our algorithms were tested on instances where disjoint paths' computation is always feasible. It would be interesting in a further work to 


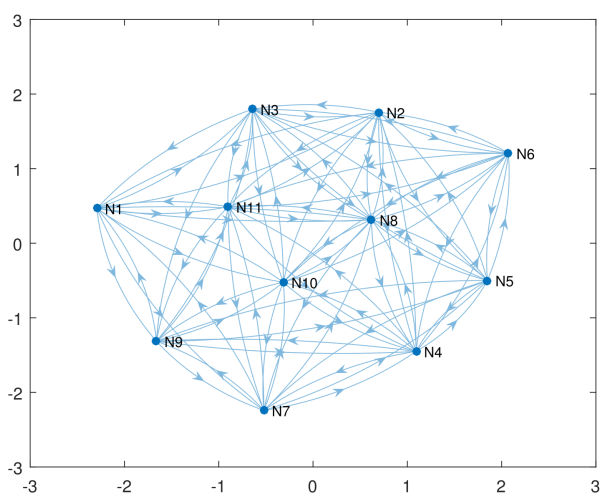

(a) Before GreenTNDP execution

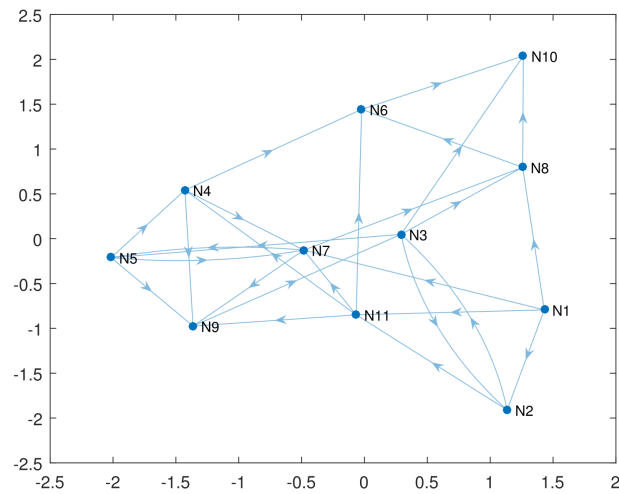

(b) After GreenTNDP execution

Fig. 4: Di-yuan network

TABLE V: Energy saving and average route reliability

\begin{tabular}{llccc}
\hline & & SP & ILP model & GreenTNDP \\
\hline Atlanta & $\eta \%$ & 80.68 & 67.61 & 73.29 \\
& ARR & 0.7379 & 0.9661 & 0.9503 \\
\hline Dfn-bwin & $\eta \%$ & 94.72 & 90.83 & 87.22 \\
& ARR & 0.9084 & 0.9646 & 0.9732 \\
\hline Di-yuan & $\eta \%$ & 94.64 & 88.39 & 91.66 \\
& ARR & 0.9365 & 0.9969 & 0.994 \\
\hline Nobel-germany & $\eta \%$ & 76.44 & 55.28 & 75 \\
& ARR & 0.8403 & 0.9418 & 0.8926 \\
\hline Pdh & $\eta \%$ & 92.27 & 88.06 & 88.06 \\
& ARR & 0.8568 & 0.9486 & 0.9486 \\
\hline Polska & $\eta \%$ & 81.94 & 71.52 & 65.97 \\
& ARR & 0.8692 & 0.8813 & 0.9923 \\
\hline
\end{tabular}

consider the case where the path-disjunction is not always feasible. The objective can hence consist in maintaining a predefined threshold of routing reliability subject to constraints that limit the number of turned-off cables.

\section{References}

[1] Yabin Ye, Felipe Jiménez Arribas, Jaafar Elmirghani, Filip Idzikowski, Jorge López Vizcaíno, Paolo Monti, Francesco Musumeci, Achille Pattavina, and Ward Van Heddeghem. Energy-efficient resilient optical networks: Challenges and tradeoffs. IEEE Communications Magazine, 53(2):144-150, 2015.

[2] Alfonso Gazo Cervero, Michele Chincoli, Lars Dittmann, Andreas Fischer, Alberto E. Garcia, Jaime Gal n-Jim nez, Laurent Lefevre, Hermann de Meer, Thierry Monteil, Paolo Monti, Anne-Cecile Orgerie, Louis-Francois Pau, Chris Phillips, Sergio
Ricciardi, Remi Sharrock, Patricia Stolf, Tuan Trinh, and Luca Valcarenghi. Green Wired Networks, pages 41-80. John Wiley \& Sons, Inc, 2015.

[3] Joseph Chabarek, Joel Sommers, Paul Barford, Cristian Estan, David Tsiang, and Steve Wright. Power awareness in network design and routing. In INFOCOM 2008. The 27th Conference on Computer Communications. IEEE. IEEE, 2008.

[4] JC Cardona Restrepo, Claus G Gruber, and C Mas Machuca. Energy profile aware routing. In 2009 IEEE International Conference on Communications Workshops, pages 1-5. IEEE, 2009.

[5] Rui Wang, Suixiang Gao, Wenguo Yang, and Zhipeng Jiang. Energy aware routing with link disjoint backup paths. Computer Networks, 115:42-53, 2017.

[6] Gongqi Lin, Sieteng Soh, and Kwan-Wu Chin. Energy-aware traffic engineering with reliability constraint. Computer Communications, 57:115-128, 2015.

[7] Gongqi Lin, Sieteng Soh, Kwan-Wu Chin, and Mihai Lazarescu. Energy aware two disjoint paths routing. Journal of Network and Computer Applications, 43:27-41, 2014.

[8] Joanna Moulierac and Truong Khoa Phan. Optimizing igp link weights for energy-efficiency in multi-period traffic matrices. Computer Communications, 61:79-89, 2015.

[9] Luca Chiaraviglio, Marco Mellia, and Fabio Neri. Minimizing isp network energy cost: formulation and solutions. IEEE/ACM Transactions on Networking (TON), 20(2):463-476, 2012

[10] Will Fisher, Martin Suchara, and Jennifer Rexford. Greening backbone networks: reducing energy consumption by shutting off cables in bundled links. In Proceedings of the first ACM SIGCOMM workshop on Green networking, pages 29-34. ACM, 2010.

[11] Ieee standard for local and metropolitan area networks-link aggregation. IEEE Std 802.1AX-2008, pages 1-163, Nov 2008.

[12] Maruti Gupta and Suresh Singh. Greening of the internet. In Proceedings of the 2003 conference on Applications, technologies, architectures, and protocols for computer communications, pages 19-26. ACM, 2003.

[13] Antonio Capone, Daniele Corti, Luca Gianoli, and Brunilde Sansó. An optimization framework for the energy management of carrier ethernet networks with multiple spanning trees. Computer Networks, 56(17):3666-3681, 2012.

[14] Ieee standards for local and metropolitan area networks virtual bridged local area networks- amendment 3: Multiple spanning trees. IEEE Std 802.1s-2002 (Amendment to IEEE Std 802.1Q, 1998 Edition), 2002

[15] Gongqi Lin, Sieteng Soh, Kwan-Wu Chin, and Mihai Lazarescu. Efficient heuristics for energy-aware routing in networks with bundled links. Computer Networks, 57(8):1774-1788, 2013.

[16] Abdelnour Aldraho and Alexander A Kist. Enabling energy efficient and resilient networks using dynamic topologies. In Sustainable Internet and ICT for Sustainability (SustainIT), 2012, pages 1-8. IEEE, 2012.

[17] Bernardetta Addis, Antonio Capone, Giuliana Carello, Luca G Gianoli, and Brunilde Sansò. On the energy cost of robustness and resiliency in ip networks. Computer Networks, 75:239-259, 2014.

[18] John W Suurballe and Robert Endre Tarjan. A quick method for finding shortest pairs of disjoint paths. Networks, 14(2):325-336, 1984.

[19] Jin Y Yen. Finding the k shortest loopless paths in a network. management Science, 17(11):712-716, 1971.

[20] Farabi Iqbal and Fernando A Kuipers. Disjoint paths in networks. Wiley Encyclopedia of Electrical and Electronics Engineering.

[21] Sebastian Orlowski, Roland Wessäly, Michal Pióro, and Artur Tomaszewski. Sndlib 1.0 - survivable network design library. Networks, 55(3):276-286, 2010.

[22] Ibm ilog cplex optimization studio. http://www.ilog.com/products/cplex/. 